\title{
Common Fixed Point Theorems in Fuzzy Metric Spaces Satisfying $\phi$-Contractive Condition with Common Limit Range Property
}

\author{
Sunny Chauhan, ${ }^{1}$ M. Alamgir Khan, ${ }^{2}$ and Wutiphol Sintunavarat ${ }^{3}$ \\ ${ }^{1}$ Near Nehru Training Centre, H. No. 274, Nai Basti B-14, Bijnor, Uttar Pradesh 246701, India \\ ${ }^{2}$ Department of Natural Resources Engineering and Management, University of Kurdistan, Hawler, Iraq \\ ${ }^{3}$ Department of Mathematics and Statistics, Faculty of Science and Technology, Thammasat University, Rangsit Center, \\ Pathum Thani 12121, Thailand
}

Correspondence should be addressed to M. Alamgir Khan; alam3333@gmail.com and Wutiphol Sintunavarat; poom_teun@hotmail.com

Received 18 June 2013; Accepted 28 July 2013

Academic Editor: Hassen Aydi

Copyright (C) 2013 Sunny Chauhan et al. This is an open access article distributed under the Creative Commons Attribution License, which permits unrestricted use, distribution, and reproduction in any medium, provided the original work is properly cited.

\begin{abstract}
The objective of this paper is to emphasize the role of "common limit range property" to ascertain the existence of common fixed point in fuzzy metric spaces. Some illustrative examples are furnished which demonstrate the validity of the hypotheses and degree of utility of our results. We derive a fixed point theorem for four finite families of self-mappings which can be utilized to derive common fixed point theorems involving any finite number of mappings. As an application to our main result, we prove an integraltype fixed point theorem in fuzzy metric space. Our results improve and extend a host of previously known results including the ones contained in Imdad et al. (2012).
\end{abstract}

\section{Introduction}

In 1965, Zadeh [1] studied the concept of a fuzzy set in his seminal paper. Thereafter, it was developed extensively by many researchers, which also include interesting applications of this theory in different fields. Fuzzy set theory has applications in applied sciences such as neural network theory, stability theory, mathematical programming, modeling theory, engineering sciences, medical sciences (medical genetics, nervous system), image processing, control theory, and communication. In 1975, Kramosil and Michálek [2] introduced the concept of fuzzy metric space, which opened an avenue for further development of analysis in such spaces. Further, George and Veeramani [3] modified the concept of fuzzy metric space introduced by Kramosil and Michálek [2] and also have succeeded in inducing a Hausdorff topology on such a fuzzy metric space which is often used in current research these days. Most recently, Gregori et al. [4] showed several interesting examples of fuzzy metrics in the sense of George and Veeramani [3] and have also utilized such fuzzy metrics to color image processing.
On the other hand, Mishra et al. [5] extended the notion of compatible mappings to fuzzy metric spaces and proved common fixed point theorems in presence of continuity of at least one of the mappings, completeness of the underlying space, and containment of the ranges amongst involved mappings. Further, Singh and Jain [6] weakened the notion of compatibility by using the notion of weakly compatible mappings in fuzzy metric spaces and showed that every pair of compatible mappings is weakly compatible, but reverse is not true. Many mathematicians used different conditions on self-mappings and proved several fixed point theorems for contractions in fuzzy metric spaces (see [6-13]). However, the study of common fixed points of noncompatible maps is also of great interest according to Pant [14]. In 2002, Aamri and El Moutawakil [15] defined a property (E.A.) for self-mappings which contained the class of noncompatible mappings in metric spaces. In a paper of Ali and Imdad [16], it was pointed out that property (E.A.) allows replacing the completeness requirement of the space with a more natural condition of closedness of the range. Afterwards, Liu et al. [17] defined a new property which contains the property 
(E.A.) and proved some common fixed point theorems under hybrid contractive conditions. It was observed that the notion of common property (E.A.) relatively relaxes the required containment of the range of one mapping into the range of other which is utilized to construct the sequence of joint iterates. Subsequently, there are a number of results proved for contraction mappings satisfying property (E.A.) and common property (E.A.) in fuzzy metric spaces (see [1825]). In 2011, Sintunavarat and Kumam [26] coined the idea of "common limit range property" (also see [27-33]) which relaxes the condition of closedness of the underlying subspace. Recently, Imdad et al. [34] extended the notion of common limit range property to two pairs of self-mappings which relaxes the requirement on closedness of the subspaces. Several common fixed point theorems have been proved by many researchers in the framework of fuzzy metric spaces via implicit relations (see $[6,22,35]$ ).

In this paper, we prove some common fixed point theorems for weakly compatible mappings with common limit range property in fuzzy metric spaces which include fuzzy metric spaces of two types, namely, Kramosil and Michálek fuzzy metric spaces and George and Veeramani fuzzy metric spaces. Some related results are also derived besides furnishing illustrative examples. We also present some integral-type common fixed point theorems in fuzzy metric spaces. Our results improve, extend, and generalize a host of previously known results existing in the literature.

\section{Preliminaries}

Definition 1 (see [36]). A binary operation $*:[0,1] \times[0,1] \rightarrow$ $[0,1]$ is said to be continuous $t$-norm if

(1) * is commutative and associative;

(2) * is continuous;

(3) $a * 1=a$ for all $a \in[0,1]$;

(4) $a * b \leq c * d$ whenever $a \leq c$ and $b \leq d$ for all $a, b, c, d \in$ $[0,1]$.

Examples of continuous $t$-norms are Lukasiewicz $t$-norm, that is, $a *_{L} b=\max \{a+b-1,0\}$, product $t$-norm, that is, $a *_{P} b=a b$, and minimum $t$-norm, that is, $a *_{M} b=$ $\min \{a, b\}$.

The fuzzy metric space of Kramosil and Michálek [2] is defined as follows.

Definition 2 (see [2]). The 3-tuple $(X, M, *)$ is said to be a KM-fuzzy metric space if $X$ is an arbitrary set, $*$ is a continuous $t$-norm, and $M$ is a fuzzy set on $X^{2} \times[0, \infty)$ satisfying the following conditions: for all $x, y, z \in X$ and $t, s>0$

$(\mathrm{KM}-1): M(x, y, 0)=0$;

(KM-2): $M(x, y, t)=1$ if and only if $x=y$;

$(\mathrm{KM}-3): M(x, y, t)=M(y, x, t)$;

(KM-4): $M(x, z, t+s) \geq M(x, y, t) * M(y, z, s)$;

$(\mathrm{KM}-5): M(x, y, \cdot):[0, \infty) \rightarrow[0,1]$ is left continuous.
Lemma 3 (see [37]). Let $(X, M, *)$ be a fuzzy metric space. Then $M(x, y, \cdot)$ is nondecreasing on $(0, \infty)$ for all $x, y \in X$.

The fuzzy metric space of George and Veeramani [3] is defined as follows.

Definition 4 (see [3]). The 3-tuple $(X, M, *)$ is said to be a GVfuzzy metric space if $X$ is an arbitrary set, $*$ is a continuous $t$-norm, and $M$ is a fuzzy set on $X^{2} \times(0, \infty)$ satisfying the following conditions: for all $x, y, z \in X$ and $t, s>0$

$$
\begin{aligned}
& (\mathrm{GV}-1): M(x, y, t)>0 ; \\
& (\mathrm{GV}-2): M(x, y, t)=1 \text { if and only if } x=y ; \\
& (\mathrm{GV}-3): M(x, y, t)=M(y, x, t) ; \\
& (\mathrm{GV}-4): M(x, z, t+s) \geq M(x, y, t) * M(y, z, s) ; \\
& (\mathrm{GV}-5): M(x, y, \cdot):(0, \infty) \rightarrow[0,1] \text { is continuous. }
\end{aligned}
$$

In view of $(\mathrm{GV}-1)$ and $(\mathrm{GV}-2)$, it is worth pointing out that $0<M(x, y, t)<1$ (for all $t>0)$ provided $x \neq y$ (see [24]).

Example 5 (see [3]). Let $(X, d)$ be a metric space. Define $M$ : $X^{2} \times(0, \infty) \rightarrow[0,1]$ as

$$
M(x, y, t)=\frac{t}{t+d(x, y)}
$$

for all $x, y \in X$ and $t>0$. Then $(X, M, *)$ is a $\mathrm{GV}$-fuzzy metric space, where $*$ is the product $t$-norm (or minimum $t$-norm). Indeed, we call this fuzzy metric $M$ induced by metric $d$ the standard fuzzy metric. Hence every metric space is a fuzzy metric space.

Now we give some examples of fuzzy metric spaces according to Gregori et al. [4].

Example 6 (see [4]). Let $X$ be a nonempty set, $f: X \rightarrow \mathbb{R}^{+}$ a one-one function, and $g: \mathbb{R}^{+} \rightarrow[0, \infty)$ an increasing continuous function. For fixed $\alpha, \beta>0$, define $M: X^{2} \times$ $(0, \infty) \rightarrow[0,1]$ as

$$
M(x, y, t)=\left(\frac{(\min \{f(x), f(y)\})^{\alpha}+g(t)}{(\max \{f(x), f(y)\})^{\alpha}+g(t)}\right)^{\beta},
$$

for all $x, y \in X$ and $t>0$. Then, $(X, M, *)$ is a fuzzy metric space on $X$ wherein $*$ is the product $t$-norm.

Example 7 (see [4]). Let $(X, d)$ be a metric space and $g$ : $\mathbb{R}^{+} \rightarrow[0, \infty)$ an increasing continuous function. Define $M: X^{2} \times(0, \infty) \rightarrow[0,1]$ as

$$
M(x, y, t)=e^{(-d(x, y) / g(t))},
$$

for all $x, y \in X$ and $t>0$. Then $(X, M, *)$ is a fuzzy metric space on $X$ wherein $*$ is the product $t$-norm.

Example 8 (see [4]). Let $(X, d)$ be a bounded metric space with $d(x, y)<k$ (for all $x, y \in X$, where $k$ is fixed constant in $(0, \infty))$ and $g: \mathbb{R}^{+} \rightarrow(k, \infty)$ an increasing continuous function. Define a function $M: X^{2} \times(0, \infty) \rightarrow[0,1]$ as

$$
M(x, y, t)=1-\frac{d(x, y)}{g(t)},
$$


for all $x, y \in X$ and $t>0$. Then $(X, M, *)$ is a fuzzy metric space on $X$ wherein $*$ is a Lukasiewicz $t$-norm.

Definition 9 (see [24]). A sequence $\left\{x_{n}\right\}$ in a KM- (or GV-) fuzzy metric space $(X, M, *)$ is said to be convergent to some $x \in X$ if for all $t>0$ there is some $n_{0} \in \mathbb{N}$ such that

$$
\lim _{n \rightarrow \infty} M\left(x_{n}, x, t\right)=1
$$

for all $n \geq n_{0}$.

Lemma 10 (see [24]). If $(X, M, *)$ is a KM-fuzzy metric space and $\left\{x_{n}\right\},\left\{y_{n}\right\}$ are sequences in $X$ such that $x_{n} \rightarrow x, y_{n} \rightarrow y$, then $M\left(x_{n}, y_{n}, t\right) \rightarrow M(x, y, t)$ for every continuity point $t$ of $M(x, y, \cdot)$.

Definition 11 (see [5]). A pair $(A, S)$ of self-mappings of a KM(or $\mathrm{GV}$-) fuzzy metric space $(X, M, *)$ is said to be compatible if for all $t>0$

$$
\lim _{n \rightarrow \infty} M\left(A S x_{n}, S A x_{n}, t\right)=1,
$$

whenever $\left\{x_{n}\right\}$ is a sequence in $X$ such that $\lim _{n \rightarrow \infty} A x_{n}=$ $\lim _{n \rightarrow \infty} S x_{n}=z$ for some $z \in X$.

Definition 12 (see [5]). A pair $(A, S)$ of self-mappings of a KM- (or GV-) fuzzy metric space $(X, M, *)$ is said to be noncompatible if there exists at least one sequence $\left\{x_{n}\right\}$ in $X$ such that $\lim _{n \rightarrow \infty} A x_{n}=\lim _{n \rightarrow \infty} S x_{n}=z$ for some $z \in X$ but $\lim _{n \rightarrow \infty} M\left(A S x_{n}, S A x_{n}, t\right) \neq 1$ or nonexistent for at least one $t>0$.

Definition 13 (see [38]). A pair $(A, S)$ of self-mappings of a nonempty set $X$ is said to be weakly compatible (or coincidentally commuting) if they commute at their coincidence points; that is, if $A z=S z$ for some $z \in X$, then $A S z=S A z$.

Remark 14 (see [38]). Two compatible self-mappings are weakly compatible, but the converse is not true. Therefore the concept of weak compatibility is more general than that of compatibility.

Definition 15 (see [18]). A pair $(A, S)$ of self-mappings of a KM- (or GV-) fuzzy metric space $(X, M, *)$ is said to satisfy the property (E.A.) if there exists a sequence $\left\{x_{n}\right\}$ in $X$ such that for all $t>0$

$$
\lim _{n \rightarrow \infty} A x_{n}=\lim _{n \rightarrow \infty} S x_{n}=z
$$

for some $z \in X$.

Note that weak compatibility and property (E.A.) are independent of each other (see [39, Examples 2.1-2.2]).

Remark 16. In view of Definition 15, a pair of noncompatible mappings of a KM- (or GV-) fuzzy metric space $(X, M, *)$ satisfies the property (E.A.), but the converse need not be true (see [39, Remark 4.8 ]).

Definition 17 (see [18]). Two pairs $(A, S)$ and $(B, T)$ of selfmappings of a KM- (or GV-) fuzzy metric space $(X, M, *)$ are said to satisfy the common property (E.A.) if there exist two sequences $\left\{x_{n}\right\},\left\{y_{n}\right\}$ in $X$ such that for all $t>0$

$$
\lim _{n \rightarrow \infty} A x_{n}=\lim _{n \rightarrow \infty} S x_{n}=\lim _{n \rightarrow \infty} B y_{n}=\lim _{n \rightarrow \infty} T y_{n}=z,
$$

for some $z \in X$.

Definition 18 (see [26]). A pair $(A, S)$ of self-mappings of a $\mathrm{KM}$ - (or GV-) fuzzy metric space $(X, M, *)$ is said to satisfy the common limit range property with respect to mapping $S$ (briefly, $\left(\mathrm{CLR}_{S}\right)$ property) if there exists a sequence $\left\{x_{n}\right\}$ in $X$ such that for all $t>0$

$$
\lim _{n \rightarrow \infty} A x_{n}=\lim _{n \rightarrow \infty} S x_{n}=z,
$$

where $z \in S(X)$.

Definition 19 (see [27]). Two pairs $(A, S)$ and $(B, T)$ of selfmappings of a KM- (or GV-) fuzzy metric space $(X, M$, *) are said to satisfy the common limit range property with respect to mappings $S$ and $T$ (briefly, $\left(\mathrm{CLR}_{S T}\right)$ property) if there exist two sequences $\left\{x_{n}\right\},\left\{y_{n}\right\}$ in $X$ such that for all $t>0$

$$
\lim _{n \rightarrow \infty} A x_{n}=\lim _{n \rightarrow \infty} S x_{n}=\lim _{n \rightarrow \infty} B y_{n}=\lim _{n \rightarrow \infty} T y_{n}=z,
$$

where $z \in S(X) \cap T(X)$.

Remark 20. If $A=B$ and $S=T$, then Definition 19 implies $\left(\mathrm{CLR}_{S}\right)$ property (that is, Definition 18$)$ according to Sintunavarat and Kumam [26].

Now we show that the $\left(\mathrm{CLR}_{S T}\right)$ property implies the common property (E.A.), but converse is not true. In this regard, see the following example.

Example 21 . Let $(X, M, *)$ be a fuzzy metric space, where $X=$ $[2,11]$, with product $t$-norm defined as $a *_{P} b=a b$ for all $a, b \in[0,1]$ and

$$
M(x, y, t)=\frac{t}{t+|x-y|},
$$

for all $t>0$ and $x, y \in X$. Define the self-mappings $A, B, S$ and $T$ by

$$
A(x)= \begin{cases}3, & \text { if } x=2, \\ 5, & \text { if } 2<x \leq 7, \\ \frac{x+1}{2}, & \text { if } x>7,\end{cases}
$$$$
B(x)= \begin{cases}6, & \text { if } x=2, \\ \frac{x+6}{2}, & \text { if } 2<x \leq 7, \\ 7, & \text { if } x>7\end{cases}
$$$$
S(x)= \begin{cases}3, & \text { if } x=2, \\ 2, & \text { if } 2<x \leq 7, \\ \frac{2 x+6}{5}, & \text { if } x>7,\end{cases}
$$$$
T(x)= \begin{cases}7, & \text { if } x=2 \\ x+2, & \text { if } 2<x \leq 7 \\ 8, & \text { if } x>7\end{cases}
$$ 
Then we have $A(X)=\{3\} \cup(4,6], B(X)=(4,13 / 2] \cup\{7\}$, $S(X)=\{2,3\} \cup(4,28 / 5]$, and $T(X)=(4,9]$.

Let us consider two sequences $\left\{x_{n}\right\}=\{7+(1 / n)\}$ and $\left\{y_{n}\right\}=\{2+(1 / n)\}$ in $X$; one can verify that

$$
\lim _{n \rightarrow \infty} A x_{n}=\lim _{n \rightarrow \infty} S x_{n}=\lim _{n \rightarrow \infty} B y_{n}=\lim _{n \rightarrow \infty} T y_{n}=4 \in X,
$$

but $4 \notin S(X) \cap T(X)$. Hence both pairs $(A, S)$ and $(B, T)$ do not satisfy the $\left(\mathrm{CLR}_{S T}\right)$ property while they satisfy the common property (E.A.).

Proposition 22. If the pairs $(A, S)$ and $(B, T)$ satisfy the common property (E.A.) and $S(X)$ and $T(X)$ are closed subsets of $X$, then the pairs also share the $\left(C L R_{S T}\right)$ property.

Definition 23 (see [40]). Let $\left\{A_{i}\right\}_{i=1}^{m}$ and $\left\{S_{k}\right\}_{k=1}^{n}$ be two families of self-mappings. The pair of families $\left(A_{1} A_{2} \cdots\right.$ $A_{m}, S_{1} S_{2} \cdots S_{k}$ ) is said to be pairwise commuting if

(1) $A_{i} A_{j}=A_{j} A_{i}$ for all $i, j \in\{1,2, \ldots, m\}$;

(2) $S_{k} S_{l}=S_{l} S_{k}$ for all $k, l \in\{1,2, \ldots, n\}$;

(3) $A_{i} S_{k}=S_{k} A_{i}$ for all $i \in\{1,2, \ldots, m\}$ and $k \in\{1,2$, $\ldots, n\}$.

\section{Main Results}

Our results involve class $\Phi$ of all mappings $\phi:[0,1] \rightarrow[0,1]$ satisfying the following properties:

$\left(\phi_{1}\right): \phi$ is continuous and nondecreasing on $[0,1]$;

$\left(\phi_{2}\right): \phi(s)>s$ for all $s \in(0,1)$.

We note that if $\phi \in \Phi$, then $\phi(1)=1$, and that $\phi(s) \geq s$ for all $s \in[0,1]$.

3.1. Fixed Point Theorems in KM-Fuzzy Metric Spaces. We begin with the following observation before proving our main result.

Lemma 24. Let $A, B, S$, and $T$ be four self-mappings of a KMfuzzy metric space $(X, M, *)$. Suppose that

(1) the pair $(A, S)($ or $(B, T))$ satisfies the $\left(C L R_{S}\right)$ (or $\left.\left(C L R_{T}\right)\right)$ property;

(2) $A(X) \subset T(X)($ or $B(X) \subset S(X))$;

(3) $T(X)($ or $S(X))$ is a closed subset of $X$;

(4) $B\left(y_{n}\right)$ converges for every sequence $\left\{y_{n}\right\}$ in $X$ whenever $T\left(y_{n}\right)$ converges (or $A\left(x_{n}\right)$ converges for every sequence $\left\{x_{n}\right\}$ in $X$ whenever $S\left(x_{n}\right)$ converges);

(5) for all $x, y \in X, x \neq y$ there exists $t>0: 0<M(x$, $y, t)<1$, for some $\phi \in \Phi$,

$M(A x, B y, t)$

$\geq \phi\left(\min \left\{\begin{array}{c}M(S x, T y, t), M(A x, S x, t), M(B y, T y, t), \\ M(A x, T y, t), M(B y, S x, t)\end{array}\right\}\right)$.
Then the pairs $(A, S)$ and $(B, T)$ satisfy the $\left(C L R_{S T}\right)$ property.

Proof. If the pair $(A, S)$ enjoys the $\left(\mathrm{CLR}_{S}\right)$ property, then there exists a sequence $\left\{x_{n}\right\}$ in $X$ such that

$$
\lim _{n \rightarrow \infty} A x_{n}=\lim _{n \rightarrow \infty} S x_{n}=z,
$$

where $z \in S(X)$. By (2), $A(X) \subset T(X)$, and for each sequence $\left\{x_{n}\right\}$, there exists a sequence $\left\{y_{n}\right\}$ in $X$ such that $A x_{n}=T y_{n}$. Therefore, due to the closedness of $T(X)$,

$$
\lim _{n \rightarrow \infty} T y_{n}=\lim _{n \rightarrow \infty} A x_{n}=z,
$$

so that $z \in T(X)$ and in all $z \in S(X) \cap T(X)$. Thus, we have $A x_{n} \rightarrow z, S x_{n} \rightarrow z$, and $T y_{n} \rightarrow z$ as $n \rightarrow \infty$. By (4), sequence $\left\{B y_{n}\right\}$ converges and in all we need to show that $B y_{n} \rightarrow z$ as $n \rightarrow \infty$. Suppose that $B y_{n} \rightarrow z^{\prime}(\neq z)$ as $n \rightarrow \infty$, and then using inequality (14) with $x=x_{n}, y=y_{n}$, we have

$$
\begin{aligned}
M & \left(A x_{n}, B y_{n}, t\right) \\
& \geq \phi\left(\min \left\{\begin{array}{c}
M\left(S x_{n}, T y_{n}, t\right), M\left(A x_{n}, S x_{n}, t\right), \\
M\left(B y_{n}, T y_{n}, t\right), \\
M\left(A x_{n}, T y_{n}, t\right), M\left(B y_{n}, S x_{n}, t\right)
\end{array}\right\}\right) .
\end{aligned}
$$

Taking the limit as $n \rightarrow \infty$ and using Lemma 10, we get

$$
\begin{aligned}
& M\left(z, z^{\prime}, t\right) \\
& \quad \geq \phi\left(\min \left\{\begin{array}{c}
M(z, z, t), M(z, z, t), M\left(z^{\prime}, z, t\right), \\
M(z, z, t), M\left(z^{\prime}, z, t\right)
\end{array}\right\}\right)
\end{aligned}
$$

or, equivalently,

$$
M\left(z, z^{\prime}, t\right) \geq \phi\left(M\left(z^{\prime}, z, t\right)\right) .
$$

As $z \neq z^{\prime}$, we have $0<M\left(z, z^{\prime}, t_{0}\right)<1$ for some $t_{0}>$ 0 . Then, in view of condition $\left(\phi_{2}\right)$, we get $\phi\left(M\left(z, z^{\prime}, t_{0}\right)\right)>$ $M\left(z, z^{\prime}, t_{0}\right)$, which is a contradiction, thereby implying $z=z^{\prime}$ which shows that the pairs $(A, S)$ and $(B, T)$ enjoy the $\left(\operatorname{CLR}_{S T}\right)$ property.

Remark 25. The converse of Lemma 24 is not true in general. For counterexamples, one can see Examples 27 and 30.

Theorem 26. Let $A, B, S$, and T be four self-mappings of a KMfuzzy metric space $(X, M, *)$ satisfying inequality (14). Suppose that the pairs $(A, S)$ and $(B, T)$ enjoy the $\left(C L R_{S T}\right)$ property. Then the pairs $(A, S)$ and $(B, T)$ have a coincidence point each. Moreover, $A, B, S$, and $T$ have a unique common fixed point provided both pairs $(A, S)$ and $(B, T)$ are weakly compatible.

Proof. Since the pairs $(A, S)$ and $(B, T)$ satisfy the $\left(\mathrm{CLR}_{S T}\right)$ property, there exist two sequences $\left\{x_{n}\right\}$ and $\left\{y_{n}\right\}$ in $X$ such that

$$
\lim _{n \rightarrow \infty} A x_{n}=\lim _{n \rightarrow \infty} S x_{n}=\lim _{n \rightarrow \infty} B y_{n}=\lim _{n \rightarrow \infty} T y_{n}=z,
$$


where $z \in S(X) \cap T(X)$. Since $z \in S(X)$, there exists a point $u \in X$ such that $S u=z$. We show that $M(A u, z, t)=1$. If not, then using inequality (14) with $x=u, y=y_{n}$, we get

$$
\begin{aligned}
& M\left(A u, B y_{n}, t\right) \\
& \quad \geq \phi\left(\min \left\{\begin{array}{c}
M\left(S u, T y_{n}, t\right), M(A u, S u, t), \\
M\left(B y_{n}, T y_{n}, t\right), \\
M\left(A u, T y_{n}, t\right), M\left(B y_{n}, S u, t\right)
\end{array}\right\}\right),
\end{aligned}
$$

which, on making $n \rightarrow \infty$ and using Lemma 10 , reduces to

$$
\begin{gathered}
M(A u, z, t) \\
\geq \phi(\min \{M(z, z, t), M(A u, z, t), M(z, z, t), \\
M(A u, z, t), M(z, z, t)\}),
\end{gathered}
$$

and so

$$
M(A u, z, t) \geq \phi(M(A u, z, t)) .
$$

If $A u \neq z$, then $0<M\left(A u, z, t_{0}\right)<1$ for some $t_{0}>0$. Then in view of condition $\left(\phi_{2}\right)$ we get $\phi\left(M\left(A u, z, t_{0}\right)\right)>$ $M\left(A u, z, t_{0}\right)$, which is a contradiction. Therefore, $A u=z$ so that $A u=z=S u$ which shows that $u$ is a coincidence point of the pair $(A, S)$.

Also $z \in T(X)$; there exists a point $v \in X$ such that $T v=z$. Now we assert that $M(z, B v, t)=1$. Assume the contrary, and then using inequality (14) with $x=u, y=v$, we have

$$
\begin{aligned}
& M(A u, B v, t) \\
& \geq \phi\left(\min \left\{\begin{array}{c}
M(S u, T v, t), M(A u, S u, t), M(B v, T v, t), \\
M(A u, T v, t), M(B v, S u, t)
\end{array}\right\}\right),
\end{aligned}
$$

which reduces to

$$
\begin{aligned}
& M(z, B v, t) \\
& \quad \geq \phi\left(\min \left\{\begin{array}{c}
M(z, z, t), M(z, z, t), M(B v, z, t), \\
M(z, z, t), M(B v, z, t)
\end{array}\right\}\right)
\end{aligned}
$$

or, equivalently,

$$
M(z, B v, t) \geq \phi(M(z, B v, t)) .
$$

As $B v \neq z$ implies $0<M\left(z, B v, t_{0}\right)<1$ for some $t_{0}>$ 0 , then in view of condition $\left(\phi_{2}\right)$, we get $\phi\left(M\left(z, B v, t_{0}\right)\right)>$ $M\left(z, B v, t_{0}\right)$, which is a contradiction. Therefore, $B v=z$ so that $B v=z=T v$ which shows that $v$ is a coincidence point of the pair $(B, T)$.

Since the pair $(A, S)$ is weakly compatible and $A u=S u$, hence $A z=A S u=S A u=S z$. Now we show that $z$ is a common fixed point of the pair $(A, S)$. To prove this, we show that $M(A z, z, t)=1$. If not, then using inequality (14) with $x=z, y=v$, we have

$$
\begin{aligned}
& M(A z, B v, t) \\
& \geq \phi\left(\min \left\{\begin{array}{c}
M(S z, T v, t), M(A z, S z, t), M(B v, T v, t), \\
M(A z, T v, t), M(B v, S z, t)
\end{array}\right\}\right),
\end{aligned}
$$

and so

$$
\begin{aligned}
& M(A z, z, t) \\
& \quad \geq \phi\left(\min \left\{\begin{array}{c}
M(A z, z, t), M(A z, A z, t), M(z, z, t), \\
M(A z, z, t), M(z, A z, t)
\end{array}\right\}\right) .
\end{aligned}
$$

Then on simplification, we obtain

$$
M(A z, z, t) \geq \phi(M(A z, z, t))
$$

Since $A z \neq z$, therefore $0<M\left(A z, z, t_{0}\right)<1$ for some $t_{0}>$ 0 . Then in view of condition $\left(\phi_{2}\right)$, we get $\phi\left(M\left(A z, z, t_{0}\right)\right)>$ $M\left(A z, z, t_{0}\right)$, which is a contradiction. Hence $A z=z=S z$. Therefore, $z$ is a common fixed point of the pair $(A, S)$.

Also the pair $(B, T)$ is weakly compatible and $B v=T v$; then $B z=B T v=T B v=T z$. To accomplish this, we assert that $M(z, B z, t)=1$. If not, then using inequality (14) with $x=u, y=z$, we have

$$
\begin{aligned}
& M(A u, B z, t) \\
& \quad \geq \phi\left(\min \left\{\begin{array}{c}
M(S u, T z, t), M(A u, S u, t) \\
M(B z, T z, t) \\
M(A u, T z, t), M(B z, S u, t)
\end{array}\right\}\right),
\end{aligned}
$$

which reduces to

$$
\begin{aligned}
& M(z, B z, t) \\
& \quad \geq \phi\left(\min \left\{\begin{array}{c}
M(z, B z, t), M(z, z, t), M(B z, B z, t), \\
M(z, B z, t), M(B z, z, t)
\end{array}\right\}\right),
\end{aligned}
$$

and so

$$
M(z, B z, t) \geq \phi(M(z, B z, t)) .
$$

If $B z \neq z$, then $0<M\left(z, B z, t_{0}\right)<1$ for some $t_{0}>0$. Then (in view of condition $\left.\left(\phi_{2}\right)\right)$ it follows that $\phi\left(M\left(z, B z, t_{0}\right)\right)>$ $M\left(z, B z, t_{0}\right)$, which is a contradiction. Therefore, $B z=z=$ $\mathrm{T} z$ which shows that $z$ is a common fixed point of the pair $(B, T)$. Uniqueness of common fixed point is an easy consequence of inequality (14) (in view of condition $\left(\phi_{2}\right)$ ).

Next, we give an example which is not applied by the results of Imdad et al. [21, Theorem 2.1] but can be applied to Theorem 26.

Example 27. Let $(X, M, *)$ be a fuzzy metric space, where $X=$ $[3,15)$, with product $t$-norm defined as $a *_{P} b=a b$ for all $a, b \in[0,1]$ and

$$
M(x, y, t)=\left(\frac{t}{t+1}\right)^{|x-y|}
$$


for all $t>0$ and $x, y \in X$. Define the self-mappings $A, B, S$, and $T$ by

$$
\begin{aligned}
& A(x)= \begin{cases}3, & \text { if } x \in\{3\} \cup(5,15), \\
3.5, & \text { if } x \in(3,5],\end{cases} \\
& B(x)= \begin{cases}3, & \text { if } x \in\{3\} \cup(5,15), \\
4, & \text { if } x \in(3,5],\end{cases} \\
& S(x)= \begin{cases}3, & \text { if } x=3, \\
9, & \text { if } x \in(3,5), \\
\frac{x+70}{25}, & \text { if } x \in(5,15),\end{cases} \\
& T(x)= \begin{cases}3, & \text { if } x=3, \\
14, & \text { if } x \in(3,5), \\
15, & \text { if } x=5, \\
\frac{x+70}{25}, & \text { if } x \in(5,15) .\end{cases}
\end{aligned}
$$

We obtain

$$
\begin{gathered}
A(X)=\{3,3.5\} \nsubseteq[3,3.4) \cup\{14,15\}=T(X), \\
B(X)=\{3,4\} \nsubseteq[3,3.4) \cup\{9,10\}=S(X) .
\end{gathered}
$$

Hence $S(X)$ and $T(X)$ are not closed subsets of $X$ and so Theorem 2.1 of Imdad et al. [21] can not be applied to this example.

Next, we choose two sequences $\left\{x_{n}\right\}=\{5+(1 / n)\},\left\{y_{n}\right\}=$ $\{3\}\left(\right.$ or $\left.\left\{x_{n}\right\}=\{3\},\left\{y_{n}\right\}=\{5+(1 / n)\}\right)$, and then clearly

$$
\begin{aligned}
\lim _{n \rightarrow \infty} A x_{n} & =\lim _{n \rightarrow \infty} S x_{n}=\lim _{n \rightarrow \infty} B y_{n}=\lim _{n \rightarrow \infty} T y_{n} \\
& =3 \in S(X) \cap T(X),
\end{aligned}
$$

which shows that both pairs $(A, S)$ and $(B, T)$ enjoy the $\left(\mathrm{CLR}_{S T}\right)$ property. By a routine calculation, one can verify inequality (14) (for all $x, y \in X$ and $t>0$ ) wherein $\phi$ is defined by $\phi(s)=\sqrt{s}$. Furthermore, we obtain that the pairs $(A, S)$ and $(B, T)$ are weakly compatible.

Therefore, all the conditions of Theorem 26 are satisfied and 3 is a unique common fixed point of $A, B, S$, and $T$ which also remains a coincidence point as well.

Now we show that the result contained in Imdad et al. [21, Theorem 2.1] can be easily obtained by Theorem 26 .

Theorem 28. Let $A, B, S$, and T be four self-mappings of a KMfuzzy metric space $(X, M, *)$ satisfying inequality (14). Suppose that the following hypotheses hold:

(1) the pairs $(A, S)$ and $(B, T)$ satisfy the common property (E.A.);

(2) $S(X)$ and $T(X)$ are closed subsets of $X$.

Then the pairs $(A, S)$ and $(B, T)$ have a coincidence point each. Moreover, $A, B, S$, and $T$ have a unique common fixed point provided both pairs $(A, S)$ and $(B, T)$ are weakly compatible.
Proof. Since the pairs $(A, S)$ and $(B, T)$ enjoy the common property (E.A.), there exist two sequences $\left\{x_{n}\right\}$ and $\left\{y_{n}\right\}$ in $X$ such that

$$
\lim _{n \rightarrow \infty} A x_{n}=\lim _{n \rightarrow \infty} S x_{n}=\lim _{n \rightarrow \infty} B y_{n}=\lim _{n \rightarrow \infty} T y_{n}=z,
$$

for some $z \in X$. Since $S(X)$ and $T(X)$ are closed subsets of $X$, hence $\lim _{n \rightarrow \infty} S x_{n}=z \in S(X)$. Therefore, there exists a point $u \in X$ such that $S u=z$. Similarly, $\lim _{n \rightarrow \infty} T y_{n}=z \in T(X)$. Therefore, there exists a point $v \in X$ such that $T v=z$. The rest of the proof runs on the lines of the proof of Theorem 26.

Theorem 29. Let $A, B, S$, and $T$ be four self-mappings of $a$ KM-fuzzy metric space $(X, M, *)$ satisfying all the hypotheses of Lemma 24. Then $A, B, S$, and $T$ have a unique common fixed point provided both pairs $(A, S)$ and $(B, T)$ are weakly compatible.

Proof. In view of Lemma 24, the pairs $(A, S)$ and $(B, T)$ enjoy the $\left(\mathrm{CLR}_{S T}\right)$ property; there exist two sequences $\left\{x_{n}\right\}$ and $\left\{y_{n}\right\}$ in $X$ such that

$$
\lim _{n \rightarrow \infty} A x_{n}=\lim _{n \rightarrow \infty} S x_{n}=\lim _{n \rightarrow \infty} T y_{n}=\lim _{n \rightarrow \infty} B y_{n}=t,
$$

where $t \in S(X) \cap T(X)$. The rest of the proof can be completed on the lines of the proof of Theorem 26. This completes the proof.

The following example demonstrates the utility of Theorem 29.

Example 30. In the setting of Example 27, replace the selfmappings $A, B, S$, and $T$ by the following besides retaining the rest:

$$
\begin{gathered}
A(x)= \begin{cases}3, & \text { if } x \in\{3\} \cup(5,15), \\
3.5, & \text { if } x \in(3,5],\end{cases} \\
B(x)= \begin{cases}3, & \text { if } x \in\{3\} \cup(5,15), \\
4, & \text { if } x \in(3,5],\end{cases} \\
S(x)= \begin{cases}3, & \text { if } x=3, \\
13, & \text { if } x \in(3,5], \\
x-2, & \text { if } x \in(5,15),\end{cases} \\
T(x)= \begin{cases}3, & \text { if } x=3, \\
14, & \text { if } x \in(3,5], \\
\frac{11 x-25}{10}, & \text { if } x \in(5,15) .\end{cases}
\end{gathered}
$$

Then we have $A(X)=\{3,3.5\} \subseteq[3,14]=T(X)$ and $B(X)=$ $\{3,4\} \subseteq[3,13]=S(X)$, whereas $S(X)$ and $T(X)$ are closed subsets of $X$. Then, like the earlier example, the pairs $(A, S)$ satisfy the $\left(\mathrm{CLR}_{S}\right)$ property and $(B, T)$ satisfy the $\left(\mathrm{CLR}_{T}\right)$ property.

It easy to calculate that inequality (14) holds wherein $\phi$ is defined by $\phi(s)=\sqrt{s}$. Moreover, the pairs $(A, S)$ and $(B, T)$ are weakly compatible.

Thus all the conditions of Theorem 29 are satisfied, and 3 is a unique common fixed point of the involved mappings $A$, $B, S$, and $T$. 
By choosing $A, B, S$, and $T$ suitably, we can derive a multitude of common fixed point theorems for a pair of mappings. As a sample, we deduce the following natural result for a pair of self-mappings.

Corollary 31. Let $A$ and $S$ be two self-mappings of a KM-fuzzy metric space $(X, M, *)$ satisfying the following conditions:

(1) the pair $(A, S)$ enjoys the $\left(C L R_{S}\right)$ property;

(2) for all $x, y \in X, x \neq y$, there exists $t>0: 0<M(x, y$, t) $<1$, for some $\phi \in \Phi$

$$
\begin{aligned}
& M(A x, A y, t) \\
& \quad \geq \phi\left(\min \left\{\begin{array}{c}
M(S x, S y, t), M(A x, S x, t), \\
M(A y, S y, t), \\
M(A x, S y, t), M(A y, S x, t)
\end{array}\right\}\right) .
\end{aligned}
$$

Then $A$ and S have a coincidence point. Moreover, if the pair $(A, S)$ is weakly compatible, then $A$ and $S$ have a unique common fixed point.

As an application of Theorem 26, we have the following result involving four finite families of self-mappings.

Theorem 32. Let $\left\{A_{i}\right\}_{i=1}^{m},\left\{B_{c}\right\}_{c=1}^{p},\left\{S_{j}\right\}_{j=1}^{n}$, and $\left\{T_{d}\right\}_{d=1}^{q}$ be four finite families of self-mappings of a KM-fuzzy metric space $(X, M, *)$ such that $A=A_{1} A_{2} \cdots A_{m}, B=B_{1} B_{2} \cdots B_{p}$, $S=S_{1} S_{2} \cdots S_{n}$, and $T=T_{1} T_{2} \cdots T_{q}$ which satisfy inequality (14). If the pairs $(A, S)$ and $(B, T)$ satisfy the $\left(C L R_{S T}\right)$ property, then $(A, S)$ and $(B, T)$ have a point of coincidence each.

Moreover, $\left\{A_{i}\right\}_{i=1}^{m},\left\{B_{c}\right\}_{c=1}^{p},\left\{S_{j}\right\}_{j=1}^{n}$, and $\left\{T_{d}\right\}_{d=1}^{q}$ have a unique common fixed point provided the pairs of families $\left(A_{1} A_{2} \cdots A_{m}, S_{1} S_{2} \cdots S_{n}\right)$ and $\left(B_{1} B_{2} \cdots B_{p}, T_{1} T_{2} \cdots T_{q}\right)$ are commute pairwise.

Proof. The proof of this theorem is similar to that of Theorem 3.1 contained in Imdad et al. [40]; hence the details are omitted.

Remark 33. Theorem 32 is a partial generalization of Theorem 26 as commutativity requirements in Theorem 32 are relatively stronger than weak compatibility used in Theorem 26

Now, we indicate that Theorem 32 can be utilized to derive common fixed point theorems for any finite number of mappings. As a sample for five mappings, we can derive the following by setting one family of two members while the remaining families contain single members:

Corollary 34. Let $A, B, R, S$, and $T$ be five self-mappings of a KM-fuzzy metric space $(X, M, *)$ satisfying the following conditions:

(1) the pairs $(A, S R)$ and $(B, T)$ share the $\left(C L R_{(S R)(T)}\right)$ property;
(2) for all $x, y \in X, x \neq y$, there exists $t>0$ : $0<M(x$, $y, t)<1$, for some $\phi \in \Phi$

$M(A x, B y, t)$

$$
\geq \phi\left(\min \left\{\begin{array}{c}
M(S R x, T y, t), M(A x, S R x, t), \\
M(B y, T y, t), \\
M(A x, T y, t), M(B y, S R x, t)
\end{array}\right\}\right) .
$$

Then the pairs $(A, S R)$ and $(B, T)$ have a coincidence point each. Moreover, $A, B, R, S$, and $T$ have a unique common fixed point provided the pairs $(A, S R)$ and $(B, T)$ commute pairwise (that is, $A S=S A, A R=R A, S R=R S$, and $B T=T B$ ).

Similarly, we can derive a common fixed point theorem for six mappings by setting two families of two members while the remaining families contain single members:

Corollary 35. Let $A, B, R, S, H$, and $T$ be six self-mappings of a KM-fuzzy metric space $(X, M, *)$ satisfying the following conditions:

(1) the pairs $(A, S R)$ and $(B, T H)$ enjoy the $\left(C L R_{(S R)(T H)}\right)$ property;

(2) for all $x, y \in X, x \neq y$, there exists $t>0: 0<$ $M(x, y, t)<1$, for some $\phi \in \Phi$

$$
\begin{aligned}
& M(A x, B y, t) \\
& \quad \geq \phi\left(\min \left\{\begin{array}{c}
M(S R x, T H y, t), M(A x, S R x, t), \\
M(B y, T H y, t), \\
M(A x, T H y, t), M(B y, S R x, t)
\end{array}\right\}\right) .
\end{aligned}
$$

Then the pairs $(A, S R)$ and $(B, T H)$ have a coincidence point each. Moreover, $A, B, R, S, H$, and $T$ have a unique common fixed point provided the pairs $(A, S R)$ and $(B, T H)$ commute pairwise (that is, $A S=S A, A R=R A, S R=R S, B T=$ $T B, B H=H B$, and $T H=H T$ )

By setting $A_{1}=A_{2}=\cdots=A_{m}=A, B_{1}=B_{2}=\cdots=$ $B_{p}=B, S_{1}=S_{2}=\cdots=S_{n}=S$, and $T_{1}=T_{2}=\cdots=T_{q}=T$ in Theorem 32, we deduce the following.

Corollary 36. Let $A, B, S$, and $T$ be four self-mappings of $a$ KM-fuzzy metric space $(X, M, *)$ such that the pairs $\left(A^{m}, S^{n}\right)$ and $\left(B^{p}, T^{q}\right)$ satisfy the $\left(C L R_{S^{n}, T^{q}}\right)$ property. Suppose that for all $x, y \in X, x \neq y$ there exists $t>0: 0<M(x, y, t)<1$, for some $\phi \in \Phi$

$$
\begin{aligned}
M & \left(A^{m} x, B^{p} y, t\right) \\
& \geq \phi\left(\min \left\{\begin{array}{c}
M\left(S^{n} x, T^{q} y, t\right), M\left(A^{m} x, S^{n} x, t\right), \\
M\left(B^{p} y, T^{q} y, t\right), \\
M\left(A^{m} x, T^{q} y, t\right), M\left(B^{p} y, S^{n} x, t\right)
\end{array}\right\}\right),
\end{aligned}
$$

where $m, n, p$, and $q$ are fixed positive integers. Then the pairs $(A, S)$ and $(B, T)$ have a point of coincidence each. Further, $A$, $B, S$, and $T$ have a unique common fixed point provided both pairs $\left(A^{m}, S^{n}\right)$ and $\left(B^{p}, T^{q}\right)$ commute pairwise. 
Remark 37. The results similar to Theorem 28, Theorem 29, Corollary 31, Corollary 34, and Corollary 35 can be outlined in respect of Theorem 32 and Corollary 36.

3.2. Grabiec-Type Fixed Point Results. Inspired by the work of Grabiec [37], we state and prove some fixed point theorems for weakly compatible mappings with common limit range property.

Lemma 38 (see [37]). Let (X, M, *) be a KM- (or GV-) fuzzy metric space. If there exists a constant $k \in(0,1)$ such that

$$
M(x, y, k t) \geq M(x, y, t)
$$

for all $x, y \in X, t>0$, then $x=y$.

Theorem 39. Let $A, B, S$, and T be four self-mappings of a KMfuzzy metric space $(X, M, *)$. Suppose that

(1) the pairs $(A, S)$ and $(B, T)$ enjoy the $\left(C L R_{S T}\right)$ property;

(2) for all $x, y \in X, t>0$ and for some $k \in(0,1)$

$$
\begin{aligned}
& M(A x, B y, k t) \\
& \quad \geq \min \left\{\begin{array}{c}
M(S x, T y, t), M(A x, S x, t), M(B y, T y, t), \\
M(A x, T y, t), M(B y, S x, t)
\end{array}\right\} .
\end{aligned}
$$

Then the pairs $(A, S)$ and $(B, T)$ have a coincidence point each. Moreover, $A, B, S$, and $T$ have a unique common fixed point provided both pairs $(A, S)$ and $(B, T)$ are weakly compatible.

Proof. If the pairs $(A, S)$ and $(B, T)$ share the $\left(\mathrm{CLR}_{S T}\right)$ property, then there exist two sequences $\left\{x_{n}\right\}$ and $\left\{y_{n}\right\}$ in $X$ such that

$$
\lim _{n \rightarrow \infty} A x_{n}=\lim _{n \rightarrow \infty} S x_{n}=\lim _{n \rightarrow \infty} B y_{n}=\lim _{n \rightarrow \infty} T y_{n}=z
$$

where $z \in S(X) \cap T(X)$. Since $z \in S(X)$, there exists a point $u \in X$ such that $S u=z$. Now we have to show that $A u=z$. On using inequality (45), we have

$$
\begin{aligned}
M & \left(A u, B y_{n}, k t\right) \\
& \geq \min \left\{\begin{array}{c}
M\left(S u, T y_{n}, t\right), M(A u, S u, t), M\left(B y_{n}, T y_{n}, t\right), \\
M\left(A u, T y_{n}, t\right), M\left(B y_{n}, S u, t\right)
\end{array}\right\} .
\end{aligned}
$$

Letting $n \rightarrow \infty$ and using Lemma 10 ,

$$
\begin{aligned}
& M(A u, z, k t) \\
& \geq \min \{M(z, z, t), M(A u, z, t), M(z, z, t), \\
&\quad M(A u, z, t), M(z, z, t)\} \\
&=\min \{1, M(A u, z, t), 1, M(A u, z, t), 1\} \\
&=M(A u, z, t) .
\end{aligned}
$$

Appealing to Lemma 38, we obtain $A u=z$ and so $A u=$ $z=S u$ which shows that $u$ is a coincidence point of the pair $(A, S)$.

Also $z \in T(X)$; there exists a point $v \in X$ such that $T v=z$. Now we have to assert that $B v=z$. On using inequality (45), we get

$$
\begin{aligned}
& M(A u, B v, k t) \\
& \quad \geq \min \left\{\begin{array}{c}
M(S u, T v, t), M(A u, S u, t), M(B v, T v, t), \\
M(A u, T v, t), M(B v, S u, t)
\end{array}\right\}
\end{aligned}
$$

or, equivalently,

$$
\begin{aligned}
& M(z, B v, k t) \geq \min \{M(z, z, t), M(z, z, t), M(B v, z, t), \\
&M(z, z, t), M(B v, z, t)\} \\
&= \min \{1,1, M(B v, z, t), 1, M(B v, z, t)\} \\
&= M(z, B v, t) .
\end{aligned}
$$

In view of Lemma 38, we have $B v=z$; that is, $B v=z=T v$ which shows that $v$ is a coincidence point of the pair $(B, T)$.

As the pair $(A, S)$ is weakly compatible and $A u=S u$, therefore $A z=A S u=S A u=S z$. Now we show that $z$ is a common fixed point of the pair $(A, S)$. To prove this, using inequality (45), we have

$$
\begin{aligned}
& M(A z, B v, k t) \\
& \quad \geq \min \left\{\begin{array}{c}
M(S z, T v, t), M(A z, S z, t), M(B v, T v, t), \\
M(A z, T v, t), M(B v, S z, t)
\end{array}\right\},
\end{aligned}
$$

which reduces to

$$
\begin{aligned}
M(A z, z, k t) \geq \min \{M(A z, z, t), M(A z, A z, t), \\
M(z, z, t), M(A z, z, t), M(z, A z, t)\} \\
=\min \{M(A z, z, t), 1,1, M(A z, z, t), \\
M(z, A z, t)\} \\
=M(A z, z, t) .
\end{aligned}
$$

Owing to Lemma 38, we get $A z=z=S z$. Therefore, $z$ is a common fixed point of the pair $(A, S)$.

Since pair $(B, T)$ is weakly compatible and $B v=T v$, hence $B z=B T v=T B v=T z$. On using inequality (45), we get

$$
\begin{aligned}
& M(A u, B z, k t) \\
& \quad \geq \min \left\{\begin{array}{c}
M(S u, T z, t), M(A u, S u, t), M(B z, T z, t), \\
M(A u, T z, t), M(B z, S u, t)
\end{array}\right\} .
\end{aligned}
$$

Then on simplification, we have

$$
M(z, B z, k t) \geq M(z, B z, t) .
$$


By Lemma 38, we obtain $B z=z=T z$ which shows that $z$ is a common fixed point of the pair $(B, T)$. Uniqueness of common fixed point is an easy consequence of the inequality (45) (in view of Lemma 38).

Remark 40. Theorem 39 improves and extends the results of Grabiec [37] and Imdad et al. [21, Theorem 2.5] and extends some relevant results contained in [16] to fuzzy metric spaces.

Remark 41. The results similar to Lemma 24, Theorem 28, Theorem 29, Theorem 32, Corollary 31, Corollary 34, Corollary 35, and Corollary 36 can be proved in view of contraction condition (45) which will generalize and extend several results from the literature. The listing of the possible corollaries are not included.

\subsection{Fixed Point Theorems in GV-Fuzzy Metric Spaces}

Lemma 42. Let $A, B, S$, and $T$ be four self-mappings of a $G V$ fuzzy metric space $(X, M, *)$ satisfying conditions (1)-(4) of Lemma 24. Suppose that for all $x, y \in X$, for some $\phi \in \Phi$, and for some $t>0$

$$
\begin{aligned}
& M(A x, B y, t) \\
& \geq \phi\left(\min \left\{\begin{array}{c}
M(S x, T y, t), M(A x, S x, t), M(B y, T y, t), \\
M(A x, T y, t), M(B y, S x, t)
\end{array}\right\}\right) .
\end{aligned}
$$

Then the pairs $(A, S)$ and $(B, T)$ satisfy the $\left(C L R_{S T}\right)$ property.

Proof. As the pair $(A, S)$ enjoys the $\left(\mathrm{CLR}_{S}\right)$ property, there exists a sequence $\left\{x_{n}\right\}$ in $X$ such that

$$
\lim _{n \rightarrow \infty} A x_{n}=\lim _{n \rightarrow \infty} S x_{n}=z
$$

where $z \in S(X)$. Since $A(X) \subset T(X)$, each sequence $\left\{x_{n}\right\}$ there exists a sequence $\left\{y_{n}\right\}$ in $X$ such that $A x_{n}=T y_{n}$. Therefore, due to the closedness of $T(X)$,

$$
\lim _{n \rightarrow \infty} T y_{n}=\lim _{n \rightarrow \infty} A x_{n}=z,
$$

so that $z \in S(X) \cap T(X)$. Thus in all we have $A x_{n} \rightarrow z$, $S x_{n} \rightarrow z$, and $T y_{n} \rightarrow z$ as $n \rightarrow \infty$. By (4) of Lemma 24, the sequence $\left\{B y_{n}\right\}$ converges and in all we need to show that $B y_{n} \rightarrow z$ as $n \rightarrow \infty$. Suppose that $B y_{n} \rightarrow z^{\prime}(\neq z)$ as $n \rightarrow$ $\infty$, and then using inequality (55) with $x=x_{n}, y=y_{n}$, we have

$$
\begin{aligned}
& M\left(A x_{n}, B y_{n}, t\right) \\
& \quad \geq \phi\left(\min \left\{\begin{array}{c}
M\left(S x_{n}, T y_{n}, t\right), M\left(A x_{n}, S x_{n}, t\right), \\
M\left(B y_{n}, T y_{n}, t\right), \\
M\left(A x_{n}, T y_{n}, t\right), M\left(B y_{n}, S x_{n}, t\right)
\end{array}\right\}\right),
\end{aligned}
$$

in which, on making $n \rightarrow \infty$, we obtain

$$
M\left(z, z^{\prime}, t\right) \geq \phi\left(M\left(z^{\prime}, z, t\right)\right) .
$$

As $z \neq z^{\prime}$ implies $0<M\left(z, z^{\prime}, t\right)<1$, henceforth $\phi(M(z$, $\left.\left.z^{\prime}, t\right)\right)>M\left(z, z^{\prime}, t\right)$, which is a contradiction, thereby implying $z=z^{\prime}$ which shows that the pairs $(A, S)$ and $(B, T)$ enjoy the $\left(\mathrm{CLR}_{S T}\right)$ property.

Theorem 43. Let $A, B, S$, and $T$ be four self-mappings of a $G V$ fuzzy metric space $(X, M, *)$ satisfying inequality (55). Suppose that the pairs $(A, S)$ and $(B, T)$ enjoy the $\left(C L R_{S T}\right)$ property. Then the pairs $(A, S)$ and $(B, T)$ have a coincidence point each. Moreover, $A, B, S$, and $T$ have a unique common fixed point provided both pairs $(A, S)$ and $(B, T)$ are weakly compatible.

Proof. If the pairs $(A, S)$ and $(B, T)$ satisfy the $\left(\mathrm{CLR}_{S T}\right)$ property, then there exist two sequences $\left\{x_{n}\right\}$ and $\left\{y_{n}\right\}$ in $X$ such that

$$
\lim _{n \rightarrow \infty} A x_{n}=\lim _{n \rightarrow \infty} S x_{n}=\lim _{n \rightarrow \infty} B y_{n}=\lim _{n \rightarrow \infty} T y_{n}=z,
$$

where $z \in S(X) \cap T(X)$. Since $z \in S(X)$, there exists a point $u \in X$ such that $S u=z$. We assert that $M(A u, z, t)=1$. Assume the contrary, and then using inequality (55) with $x=$ $u, y=y_{n}$, we get

$$
\begin{aligned}
M & \left(A u, B y_{n}, t\right) \\
& \geq \phi\left(\min \left\{\begin{array}{c}
M\left(S u, T y_{n}, t\right), M(A u, S u, t), \\
M\left(B y_{n}, T y_{n}, t\right), \\
M\left(A u, T y_{n}, t\right), M\left(B y_{n}, S u, t\right)
\end{array}\right\}\right),
\end{aligned}
$$

which, on making $n \rightarrow \infty$, reduces to

$$
M(A u, z, t) \geq \phi(M(A u, z, t)) .
$$

As $A u \neq z$ implies $0<M(A u, z, t)<1$, henceforth $\phi(M(A u, z, t))>M(A u, z, t)$, which is a contradiction. Therefore, $A u=z$ so that $A u=z=S u$. Hence $u$ is a coincidence point of the pair $(A, S)$.

Also $z \in T(X)$ there exists a point $v \in X$ such that $T v=z$. Now we show that $M(z, B v, t)=1$. If not, then using inequality (55) with $x=u, y=v$, we have

$$
\begin{aligned}
& M(A u, B v, t) \\
& \quad \geq \phi\left(\min \left\{\begin{array}{c}
M(S u, T v, t), M(A u, S u, t), \\
M(B v, T v, t), \\
M(A u, T v, t), M(B v, S u, t)
\end{array}\right\}\right),
\end{aligned}
$$

which reduces to

$$
M(z, B v, t) \geq \phi(M(z, B v, t)) .
$$

As $B v \neq z$ implies $0<M\left(z, B v, t_{0}\right)<1$, henceforth $\phi(M(z, B v, t))>M(z, B v, t)$, which is a contradiction. Therefore, $B v=z$ so that $B v=z=T v$ which shows that $v$ is a coincidence point of the pair $(B, T)$.

Since the pair $(A, S)$ is weakly compatible and $A u=S u$, hence $A z=A S u=S A u=S z$. Now we show that $z$ is a common fixed point of the pair $(A, S)$. To prove this, we show 
that $M(A z, z, t)=1$. Assume the contrary, and then using inequality (55) with $x=z, y=v$, we have

$M(A z, B v, t)$

$$
\geq \phi\left(\min \left\{\begin{array}{c}
M(S z, T v, t), M(A z, S z, t), \\
M(B v, T v, t), \\
M(A z, T v, t), M(B v, S z, t)
\end{array}\right\}\right) .
$$

Then on simplification, we obtain

$$
M(A z, z, t) \geq \phi(M(A z, z, t)) .
$$

As $A z \neq z$ implies $0<M\left(A z, z, t_{0}\right)<1$, henceforth $\phi(M(A z, z, t))>M(A z, z, t)$, which is a contradiction. Hence $A z=z=S z$. Therefore, $z$ is a common fixed point of the pair $(A, S)$.

As the pair $(B, T)$ is weakly compatible and $B v=T v$, then $B z=B T v=T B v=T z$. To accomplish this, we assert that $M(z, B z, t)=1$. If not, then using inequality (55) with $x=u$, $y=z$, we have

$$
\begin{aligned}
& M(A u, B z, t) \\
& \quad \geq \phi\left(\min \left\{\begin{array}{c}
M(S u, T z, t), M(A u, S u, t), \\
M(B z, T z, t), \\
M(A u, T z, t), M(B z, S u, t)
\end{array}\right\}\right),
\end{aligned}
$$

and so

$$
M(z, B z, t) \geq \phi(M(z, B z, t)) .
$$

As $B z \neq z$ implies $0<M(z, B z, t)<1$, henceforth $\phi(M(z, B z, t))>M(z, B z, t)$, which is a contradiction. Therefore $M(z, B z, t)=1$ so that $B z=z=T z$ which shows that $z$ is a common fixed point of the pair $(B, T)$. Uniqueness of common fixed point is an easy consequence of the inequality (55) (in view of condition $\left(\phi_{2}\right)$ ).

Remark 44. The results similar to Theorem 28, Theorem 29, Theorem 32, Corollary 31, Corollary 34, Corollary 35, and Corollary 36 can be proved in view of contraction condition (55) (in respect of GV-fuzzy metric spaces) which will generalize and extend several results from the literature, but due to paucity of the space we have not opted to include the details.

\section{Integral Analogue of \\ Related Fixed Point Theorems}

Branciari [41] firstly states and proves an integral-type fixed point theorem which generalized the well-known Banach Contraction Principle. Since then, many researchers have extensively proved several common fixed point theorems satisfying integral-type contractive conditions (e.g., [19, 42$46]$ ). In this section, we state and prove an integral analogue of Theorem 26.

In this section, first we state and prove an integral analogue of Theorem 26 as follows.
Theorem 45. Let $A, B, S$, and T be four self-mappings of a KMfuzzy metric space $(X, M, *)$ such that for all $x, y \in X, x \neq y$ there exists $t>0: 0<M(x, y, t)<1$, for some $\phi \in \Phi$

$$
\begin{gathered}
\int_{0}^{M(A x, B y, t)} \varphi(s) d s \geq \phi\left(\int_{0}^{m(x, y)} \varphi(s) d s\right), \\
m(x, y) \\
=\min \left\{\begin{array}{c}
M(S x, T y, t), M(A x, S x, t), M(B y, T y, t), \\
M(A x, T y, t), M(B y, S x, t)
\end{array}\right\},
\end{gathered}
$$

where $\varphi:[0, \infty) \rightarrow[0, \infty)$ is a summable non-negative Lebesgue integrable function such that

$$
\int_{\epsilon}^{1} \varphi(s) d s>0
$$

for each $\epsilon \in[0,1)$. Suppose that the pairs $(A, S)$ and $(B, T)$ satisfy the $\left(C L R_{S T}\right)$ property. Then the pairs $(A, S)$ and $(B, T)$ have a coincidence point each. Moreover, $A, B, S$, and T have a unique common fixed point provided both pairs $(A, S)$ and $(B, T)$ are weakly compatible.

Proof. Since the pairs $(A, S)$ and $(B, T)$ enjoy the $\left(\mathrm{CLR}_{S T}\right)$ property, there exist two sequences $\left\{x_{n}\right\}$ and $\left\{y_{n}\right\}$ in $X$ such that

$$
\lim _{n \rightarrow \infty} A x_{n}=\lim _{n \rightarrow \infty} S x_{n}=\lim _{n \rightarrow \infty} B y_{n}=\lim _{n \rightarrow \infty} T y_{n}=z
$$

where $z \in S(X) \cap T(X)$. Since $z \in S(X)$, there exists a point $u \in X$ such that $S u=z$. We show that $A u=z$. If not, then using inequality (69) with $x=u, y=y_{n}$, we get

$$
\int_{0}^{M\left(A u, B y_{n}, t\right)} \varphi(s) d s \geq \phi\left(\int_{0}^{m\left(u, y_{n}\right)} \varphi(s) d s\right),
$$

where

$$
\begin{aligned}
& m\left(u, y_{n}\right) \\
& =\min \left\{\begin{array}{c}
M\left(S u, T y_{n}, t\right), M(A u, S u, t), \\
M\left(B y_{n}, T y_{n}, t\right) \\
M\left(A u, T y_{n}, t\right), M\left(B y_{n}, S u, t\right)
\end{array}\right\} .
\end{aligned}
$$

Taking the limit as $n \rightarrow \infty$ in (73) and using Lemma 10, we have

$$
\begin{aligned}
\lim _{n \rightarrow \infty} \int_{0}^{M\left(A u, B y_{n}, t\right)} \varphi(s) d s & \geq \lim _{n \rightarrow \infty} \phi\left(\int_{0}^{m\left(u, y_{n}\right)} \varphi(s) d s\right), \\
\int_{0}^{M(A u, z, t)} \varphi(s) d s & \geq \phi\left(\lim _{n \rightarrow \infty} \int_{0}^{m\left(u, y_{n}\right)} \varphi(s) d s\right) \\
& =\phi\left(\int_{0}^{\lim _{n \rightarrow \infty} m\left(u, y_{n}\right)} \varphi(s) d s\right),
\end{aligned}
$$


where

$$
\begin{aligned}
& \lim _{n \rightarrow \infty} m\left(u, y_{n}\right) \\
& =\lim _{n \rightarrow \infty} \min \left\{\begin{array}{c}
M\left(S u, T y_{n}, t\right), M(A u, S u, t), \\
M\left(B y_{n}, T y_{n}, t\right), \\
M\left(A u, T y_{n}, t\right), M\left(B y_{n}, S u, t\right)
\end{array}\right\} \\
& =\min \left\{\begin{array}{c}
M(z, z, t), M(A u, z, t), M(z, z, t), \\
M(A u, z, t), M(z, z, t)
\end{array}\right\} \\
& =M(A u, z, t) .
\end{aligned}
$$

Hence from (75), we obtain

$$
\int_{0}^{M(A u, z, t)} \varphi(s) d s \geq \phi\left(\int_{0}^{M(A u, z, t)} \varphi(s) d s\right) .
$$

Since $A u \neq z$, therefore $0<M\left(A u, z, t_{0}\right)<1$ for some $t_{0}>0$. Then in view of condition $\left(\phi_{2}\right)$ we get

$$
\phi\left(\int_{0}^{M\left(A u, z, t_{0}\right)} \varphi(s) d s\right)>\int_{0}^{M\left(A u, z, t_{0}\right)} \varphi(s) d s,
$$

which is a contradiction. Therefore, $z=A u$ so that $A u=z=$ $S u$. This shows that $u$ is a coincidence point of the pair $(A, S)$.

Also $z \in T(X)$; there exists a point $v \in X$ such that $T v=z$. Now we assert that $B v=z$. If not, then using inequality (69) with $x=u, y=v$, we have

$$
\int_{0}^{M(A u, B v, t)} \varphi(s) d s \geq \phi\left(\int_{0}^{m(u, v)} \varphi(s) d s\right),
$$

where

$$
\begin{aligned}
m(u, v) & \\
& =\min \left\{\begin{array}{c}
M(S u, T v, t), M(A u, S u, t), M(B v, T v, t), \\
M(A u, T v, t), M(B v, S u, t)
\end{array}\right\} \\
& =\min \left\{\begin{array}{c}
M(z, z, t), M(z, z, t), M(B v, z, t), \\
M(z, z, t), M(B v, z, t)
\end{array}\right\} \\
& =M(z, B v, t) .
\end{aligned}
$$

From (79), we get

$$
\int_{0}^{M(z, B v, t)} \varphi(s) d s \geq \phi\left(\int_{0}^{M(z, B v, t)} \varphi(s) d s\right) .
$$

As $B v \neq z$, then $0<M\left(z, B v, t_{0}\right)<1$ for some $t_{0}>0$. As $M(z, B v, \cdot)$ is left-continuous and the $M(z, B v, \cdot)$ is nondecreasing, it has only (at most) countable points of discontinuity. Now, one may suppose that $t_{0}$ is a continuity point of $M(z, B v, \cdot)$, and then (in view of condition $\left(\phi_{2}\right)$ ) we get

$$
\phi\left(\int_{0}^{M\left(z, B v, t_{0}\right)} \varphi(s) d s\right)>\int_{0}^{M\left(z, B v, t_{0}\right)} \varphi(s) d s,
$$

which is a contradiction. Therefore, $z=B v$ so that $B v=z=$ $T v$. Hence $v$ is a coincidence point of the pair $(B, T)$.
As the pair $(A, S)$ is weakly compatible and $A u=S u$, then $A z=A S u=S A u=S z$. Now we show that $z$ is a common fixed point of the pair $(A, S)$. To prove this, we show that $A z=$ $z$. If not, then using inequality (69) with $x=z, y=v$, we have

$$
\int_{0}^{M(A z, B v, t)} \varphi(s) d s \geq \phi\left(\int_{0}^{m(z, v)} \varphi(s) d s\right),
$$

where

$$
\begin{aligned}
m & (z, v) \\
& =\min \left\{\begin{array}{c}
M(S z, T v, t), M(A z, S z, t), M(B v, T v, t), \\
M(A z, T v, t), M(B v, S z, t)
\end{array}\right\} \\
& =\min \left\{\begin{array}{c}
M(A z, z, t), M(A z, A z, t), M(z, z, t), \\
M(A z, z, t), M(z, A z, t)
\end{array}\right\} \\
& =M(A z, z, t) .
\end{aligned}
$$

Hence (83) implies

$$
\int_{0}^{M(A z, z, t)} \varphi(s) d s \geq \phi\left(\int_{0}^{M(A z, z, t)} \varphi(s) d s\right) .
$$

If $A z \neq z$, then $0<M\left(A z, z, t_{0}\right)<1$ for some $t_{0}>0$. As $M(A z, z, \cdot)$ is left-continuous and $M(A z, z, \cdot)$ is nondecreasing, it has only (at most) countable points of discontinuity. If we suppose that $t_{0}$ is a continuity point of $M(A z, z, \cdot)$, then (in view of condition $\left(\phi_{2}\right)$ ) it follows that

$$
\phi\left(\int_{0}^{M\left(A z, z, t_{0}\right)} \varphi(s) d s\right)>\int_{0}^{M\left(A z, z, t_{0}\right)} \varphi(s) d s,
$$

which is a contradiction. Therefore, $A z=z=B z$ which shows that $z$ is a common fixed point of the pair $(A, S)$.

Since the pair $(B, T)$ is weakly compatible and $B v=T v$, $B z=B T v=T B v=T z$. To prove this, we assert that $B z=z$. If not, then using inequality (69) with $x=u, y=z$, we have

$$
\int_{0}^{M(A u, B z, t)} \varphi(s) d s \geq \phi\left(\int_{0}^{m(u, z)} \varphi(s) d s\right),
$$

where

$$
\begin{aligned}
m(u, z) & \\
& =\min \left\{\begin{array}{c}
M(S u, T z, t), M(A u, S u, t), M(B z, T z, t), \\
M(A u, T z, t), M(B z, S u, t)
\end{array}\right\} \\
& =\min \left\{\begin{array}{c}
M(z, B z, t), M(z, z, t), M(B z, B z, t), \\
M(z, B z, t), M(B z, z, t)
\end{array}\right\} \\
& =M(z, B z, t) .
\end{aligned}
$$

From (87), we get

$$
\int_{0}^{M(z, B z, t)} \varphi(s) d s \geq \phi\left(\int_{0}^{M(z, B z, t)} \varphi(s) d s\right) .
$$


As earlier, we obtain $B z=z=T z$ which shows that $z$ is a common fixed point of the pair $(B, T)$. Hence $z$ is a common fixed point of $A, B, S$, and $T$. Uniqueness of the common fixed point is an easy consequence of condition (69) in respect of condition $\left(\phi_{2}\right)$. This concludes the proof.

Now we state the earlier proved results (Theorems 39 and 43) in the framework of integral settings.

Motivated by the results of Altun et al. [47], we need the following lemma to prove Corollary 47.

Lemma 46 (see [19]). Let (X, M, *) be a KM- (or GV-) fuzzy metric space. If there exists a constant $k \in(0,1)$ such that for all $x, y \in X$ and all $t>0$

$$
\int_{0}^{M(x, y, k t)} \varphi(s) d s \geq \int_{0}^{M(x, y, t)} \varphi(s) d s,
$$

where $\varphi:[0, \infty) \rightarrow[0, \infty)$ is a summable non-negative Lebesgue integrable function such that

$$
\int_{\epsilon}^{1} \varphi(s) d s>0,
$$

for each $\epsilon \in[0,1)$, and then $x=y$.

Corollary 47. Let $A, B, S$, and $T$ be four self-mappings of a KM-fuzzy metric space $(X, M, *)$ such that for all $x, y \in X$, $t>0$ and for some $k \in(0,1)$

$$
\begin{gathered}
\int_{0}^{M(A x, B y, k t)} \varphi(s) d s \geq \int_{0}^{m(x, y)} \varphi(s) d s, \\
\quad=\min \left\{\begin{array}{c}
M(S x, T y, t), M(A x, S x, t), M(B y, T y, t), \\
M(A x, T y, t), M(B y, S x, t)
\end{array}\right\},
\end{gathered}
$$

where $\varphi:[0, \infty) \rightarrow[0, \infty)$ is a summable non-negative Lebesgue integrable function such that

$$
\int_{\epsilon}^{1} \varphi(s) d s>0,
$$

for each $\epsilon \in[0,1)$. Suppose that the pairs $(A, S)$ and $(B, T)$ satisfy the $\left(C L R_{S T}\right)$ property. Then the pairs $(A, S)$ and $(B, T)$ have a coincidence point each. Moreover, $A, B, S$, and $T$ have a unique common fixed point provided both pairs $(A, S)$ and $(B, T)$ are weakly compatible.

Corollary 48. Let $A, B, S$, and $T$ be four self-mappings of a $G V$-fuzzy metric space $(X, M, *)$ such that for all $x, y \in X$, for some $\phi \in \Phi$, and for some $t>0$

$$
\begin{aligned}
& \int_{0}^{M(A x, B y, t)} \varphi(s) d s \geq \phi\left(\int_{0}^{m(x, y)} \varphi(s) d s\right), \\
& m(x, y) \\
& =\min \left\{\begin{array}{c}
M(S x, T y, t), M(A x, S x, t), M(B y, T y, t), \\
M(A x, T y, t), M(B y, S x, t)
\end{array}\right\},
\end{aligned}
$$

where $\varphi:[0, \infty) \rightarrow[0, \infty)$ is a summable non-negative Lebesgue integrable function such that

$$
\int_{\epsilon}^{1} \varphi(s) d s>0
$$

for each $\epsilon \in[0,1)$. Suppose that the pairs $(A, S)$ and $(B, T)$ satisfy the $\left(C L R_{S T}\right)$ property. Then the pairs $(A, S)$ and $(B, T)$ have a coincidence point each. Moreover, $A, B, S$, and $T$ have a unique common fixed point provided both pairs $(A, S)$ and $(B, T)$ are weakly compatible.

Remark 49. Theorem 45 improves and generalizes the results of Miheţ [24], Imdad et al. [21], Shao and $\mathrm{Hu}$ [44, Theorem 3.2], and Murthy et al. [42, Theorems 2, 3, 5] and extend the result of Sedghi and Shobe [43, Theorem 2.2].

\section{Acknowledgments}

The authors would like to express their sincere thanks to the anonymous referees for reading the manuscript very carefully. The first author is grateful to Dr. Javid Ali for the reprint of his valuable paper [21].

\section{References}

[1] L. A. Zadeh, "Fuzzy sets," Information and Computation, vol. 8, pp. 338-353, 1965.

[2] I. Kramosil and J. Michálek, "Fuzzy metrics and statistical metric spaces," Kybernetika, vol. 11, no. 5, pp. 336-344, 1975.

[3] A. George and P. Veeramani, "On some results in fuzzy metric spaces," Fuzzy Sets and Systems, vol. 64, no. 3, pp. 395-399, 1994.

[4] V. Gregori, S. Morillas, and A. Sapena, "Examples of fuzzy metrics and applications," Fuzzy Sets and Systems, vol. 170, pp. 95111, 2011.

[5] S. N. Mishra, N. Sharma, and S. L. Singh, "Common fixed points of maps on fuzzy metric spaces," International Journal of Mathematics and Mathematical Sciences, vol. 17, no. 2, pp. 253-258, 1994.

[6] B. Singh and S. Jain, "Semicompatibility and fixed point theorems in fuzzy metric space using implicit relation," International Journal of Mathematics and Mathematical Sciences, no. 16, pp. 2617-2629, 2005.

[7] P. Balasubramaniam, S. Muralisankar, and R. P. Pant, "Common fixed points of four mappings in a fuzzy metric space," Journal of Fuzzy Mathematics, vol. 10, no. 2, pp. 379-384, 2002.

[8] I. Beg, S. Sedghi, and N. Shobe, "Fixed point theorems in fuzzy metric spaces," International Journal of Analysis, vol. 2013, Article ID 934145, 4 pages, 2013.

[9] S. Chauhan and B. D. Pant, "Common fixed point theorems in fuzzy metric spaces," Bulletin of the Allahabad Mathematical Society, vol. 27, no. 1, pp. 27-43, 2012.

[10] Y. J. Cho, "Fixed points in fuzzy metric spaces," Journal of Fuzzy Mathematics, vol. 5, no. 4, pp. 949-962, 1997.

[11] M. Imdad and J. Ali, "Some common fixed point theorems in fuzzy metric spaces," Mathematical Communications, vol. 11, no. 2, pp. 153-163, 2006.

[12] D. O'Regan and M. Abbas, "Necessary and sufficient conditions for common fixed point theorems in fuzzy metric space," Demonstratio Mathematica, vol. 42, no. 4, pp. 887-900, 2009. 
[13] S. Sharma, "Common fixed point theorems in fuzzy metric spaces," Fuzzy Sets and Systems. An International Journal in Information Science and Engineering, vol. 127, no. 3, pp. 345-352, 2002.

[14] R. P. Pant, "Common fixed points of four mappings," Bulletin of the Calcutta Mathematical Society, vol. 90, no. 4, pp. 281-286, 1998.

[15] M. Aamri and D. El Moutawakil, "Some new common fixed point theorems under strict contractive conditions," Journal of Mathematical Analysis and Applications, vol. 270, no. 1, pp. 181188, 2002.

[16] J. Ali and M. Imdad, "An implicit function implies several contraction conditions," Sarajevo Journal of Mathematics, vol. 4, no. 4, pp. 269-285, 2008.

[17] Y. Liu, J. Wu, and Z. Li, "Common fixed points of single-valued and multivalued maps," International Journal of Mathematics and Mathematical Sciences, no. 19, pp. 3045-3055, 2005.

[18] M. Abbas, I. Altun, and D. Gopal, "Common fixed point theorems for non compatible mappings in fuzzy metric spaces," Bulletin of Mathematical Analysis and Applications, vol. 1, no. 2, pp. 47-56, 2009.

[19] S. Chauhan and S. Kumar, "Coincidence and fixed points in fuzzy metrtc spaces using common property (E.A)," Kochi Journal of Mathematics, vol. 8, pp. 135-154, 2013.

[20] D. Gopal, M. Imdad, and C. Vetro, "Impact of common property (E.A.) on fixed point theorems in fuzzy metric spaces," Fixed Point Theory and Applications, vol. 2011, Article ID 297360, 14 pages, 2011.

[21] M. Imdad, J. Ali, and M. Hasan, "Common fixed point theorems in fuzzy metric spaces employing common property (E.A.)," Mathematical and Computer Modelling, vol. 55, no. 3-4, pp. 770778, 2012.

[22] S. Kumar and B. Fisher, "A common fixed point theorem in fuzzy metric space using property (E.A.) and implicit relation," Thai Journal of Mathematics, vol. 8, no. 3, pp. 439-446, 2010.

[23] S. Kumar and S. Chauhan, "Common fixed point theorems using implicit relation and property (E.A) in fuzzy metric spaces," Annals of Fuzzy Mathematics and Informatics, vol. 5, no. 1, pp. 107-114, 2013.

[24] D. Miheț, "Fixed point theorems in fuzzy metric spaces using property (E.A)," Nonlinear Analysis. Theory, Methods \& Applications, vol. 73, no. 7, pp. 2184-2188, 2010.

[25] V. Pant and R. P. Pant, "Fixed points in fuzzy metric space for noncompatible maps," Soochow Journal of Mathematics, vol. 33, no. 4, pp. 647-655, 2007.

[26] W. Sintunavarat and P. Kumam, "Common fixed point theorems for a pair of weakly compatible mappings in fuzzy metric spaces," Journal of Applied Mathematics, vol. 2011, Article ID 637958, 14 pages, 2011.

[27] S. Chauhan, "Fixed points of weakly compatible mappings in fuzzy metric spaces satisfying common limit in the range property," Indian Journal of Mathematics, vol. 54, no. 3, pp. 375-397, 2012.

[28] S. Chauhan, S. Bhatnagar, and S. Radenović, "Common fixed point theorems for weakly compatible mappings in fuzzy metric spaces," Le Matematiche, vol. 68, no. 1, pp. 87-98, 2013.

[29] S. Chauhan, M. A. Khan, and S. Kumar, "Unified fixed point theorems in fuzzy metric spaces via common limit range property," Journal of Inequalities and Applications, vol. 2013, article 182, 2013.
[30] S. Chauhan, W. Sintunavarat, and P. Kumam, "Common fixed point theorems for weakly compatible mappings in fuzzy metric spaces using (JCLR) property," Applied Mathematics, vol. 3, no. 9, pp. 976-982, 2012.

[31] S. L. Singh, B. D. Pant, and S. Chauhan, "Fixed point theorems in non-Archimedean Menger PM-spaces," Journal of Nonlinear Analysis and Optimization, vol. 3, no. 2, pp. 153-160, 2012.

[32] W. Sintunavarat and P. Kumam, "Common fixed points for $R$ weakly commuting in fuzzy metric spaces," Annali dell'Universitá di Ferrara, vol. 58, no. 2, pp. 389-406, 2012.

[33] W. Sintunavarat, S. Chauhan, and P. Kumam, "Some fixed point results in modified intuitionistic fuzzy metric spaces," Afrika Matematika. In press.

[34] M. Imdad, B. D. Pant, and S. Chauhan, "Fixed point theorems in Menger spaces using the $\left(C L R_{S T}\right)$ property and applications," Journal of Nonlinear Analysis and Optimization, vol. 3, no. 2, pp. 225-237, 2012.

[35] M. Imdad and J. Ali, "A general fixed point theorem in fuzzy metric spaces via an implicit function," Journal of Applied Mathematics \& Informatics, vol. 26, no. 3-4, pp. 591-603, 2008.

[36] B. Schweizer and A. Sklar, Probabilistic Metric Spaces, NorthHolland Series in Probability and Applied Mathematics, NorthHolland, New York, NY, USA, 1983.

[37] M. Grabiec, "Fixed points in fuzzy metric spaces," Fuzzy Sets and Systems, vol. 27, no. 3, pp. 385-389, 1988.

[38] G. Jungck, "Common fixed points for noncontinuous nonself maps on nonmetric spaces," Far East Journal of Mathematical Sciences, vol. 4, no. 2, pp. 199-215, 1996.

[39] H. K. Pathak, R. Rodríguez-López, and R. K. Verma, "A common fixed point theorem using implicit relation and property (E.A) in metric spaces," Filomat, vol. 21, no. 2, pp. 211-234, 2007.

[40] M. Imdad, J. Ali, and M. Tanveer, "Coincidence and common fixed point theorems for nonlinear contractions in Menger PM spaces," Chaos, Solitons \& Fractals, vol. 42, no. 5, pp. 3121-3129, 2009.

[41] A. Branciari, "A fixed point theorem for mappings satisfying a general contractive condition of integral type," International Journal of Mathematics and Mathematical Sciences, vol. 29, no. 9, pp. 531-536, 2002.

[42] P. P. Murthy, S. Kumar, and K. Tas, "Common fixed points of self maps satisfying an integral type contractive condition in fuzzy metric spaces," Mathematical Communications, vol. 15, no. 2, pp. 521-537, 2010.

[43] S. Sedghi and N. Shobe, "Common fixed point theorems for weakly compatible mappings satisfying contractive condition of integral type," Journal of Advanced Research in Applied Mathematics, vol. 3, no. 4, pp. 67-78, 2011.

[44] X. Shao and X. Hu, "Common fixed point under integral type contractive condition in fuzzy metric spaces," in Proceedings of the 3rd International Workshop on Advanced Computational Intelligence (IWACI '10), pp. 131-134, Jiangsu, China, August 2010.

[45] W. Sintunavarat and P. Kumam, "Gregus-type common fixed point theorems for tangential multivalued mappings of integral type in metric spaces," International Journal of Mathematics and Mathematical Sciences, vol. 2011, Article ID 923458, 12 pages, 2011.

[46] W. Sintunavarat and P. Kumam, "Gregus type fixed points for a tangential multi-valued mappings satisfying contractive conditions of integral type," Journal of Inequalities and Applications, vol. 2011, article 3, 2011. 
[47] I. Altun, M. Tanveer, and M. Imdad, "Common fixed point theorems of integral type in Menger PM spaces," Journal of Nonlinear Analysis and Optimization, vol. 3, no. 1, pp. 55-66, 2012. 


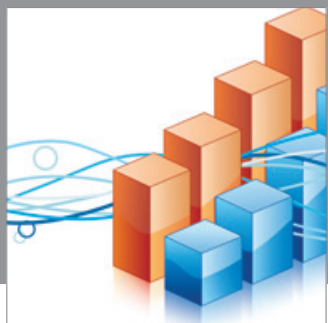

Advances in

Operations Research

mansans

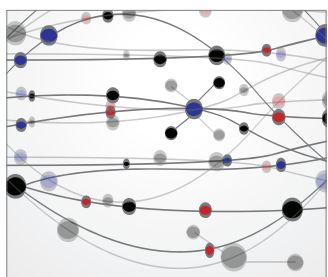

The Scientific World Journal
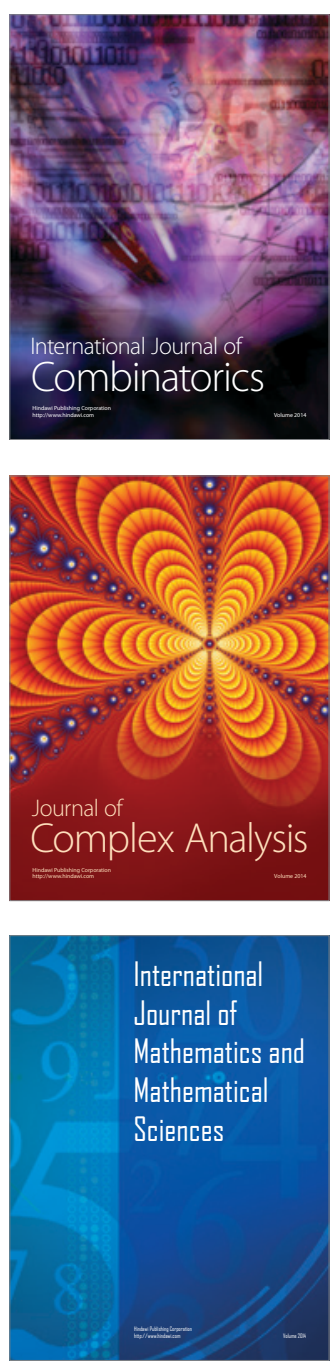
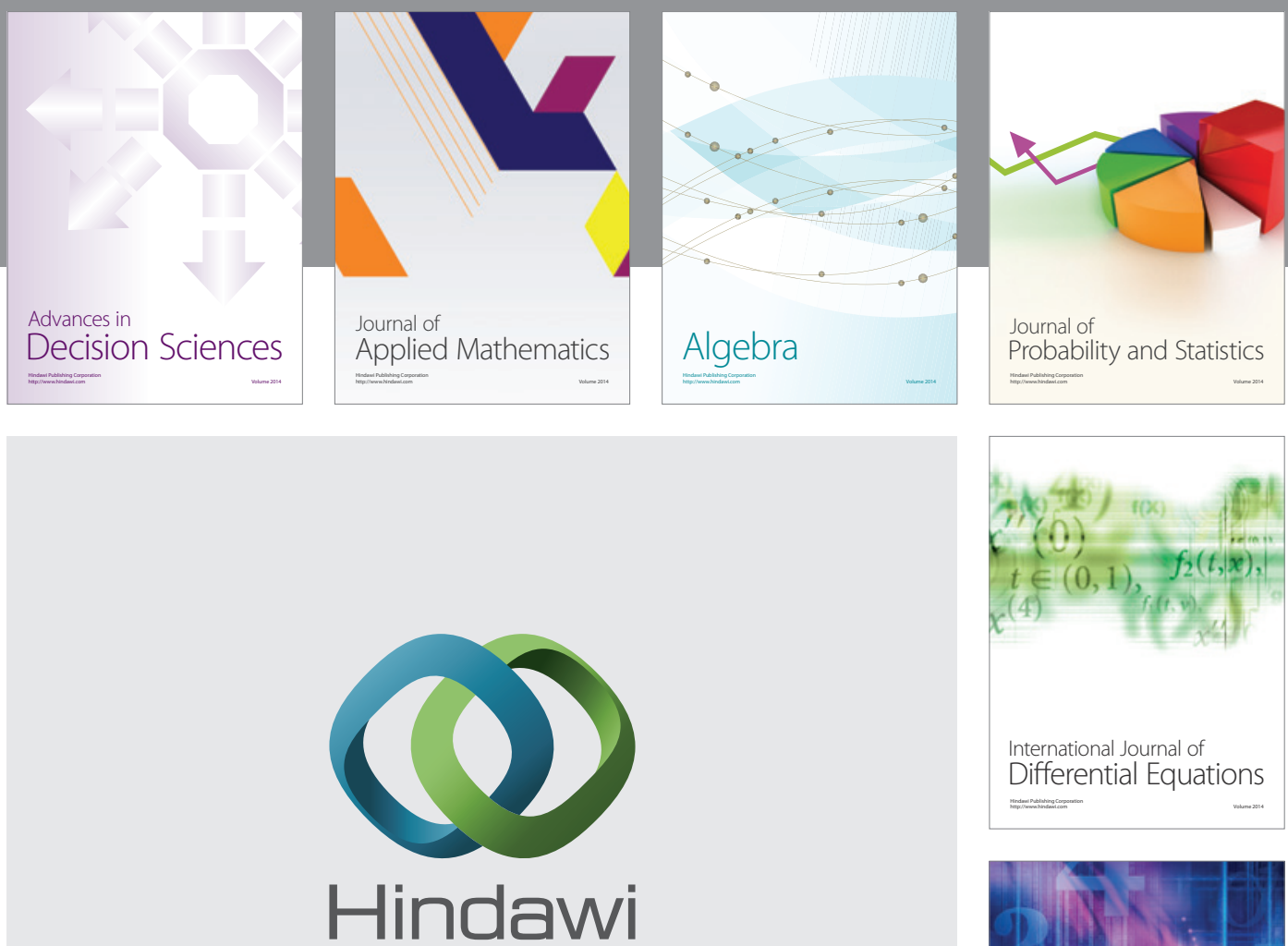

Submit your manuscripts at http://www.hindawi.com
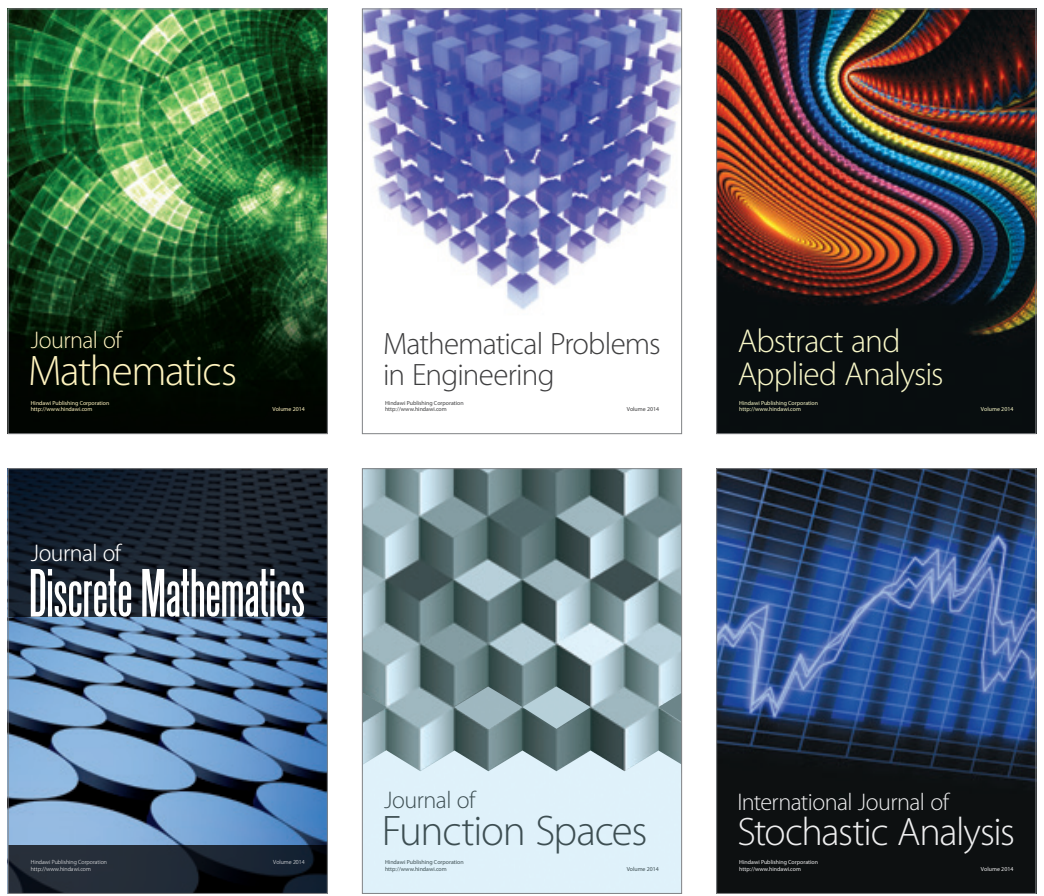

Journal of

Function Spaces

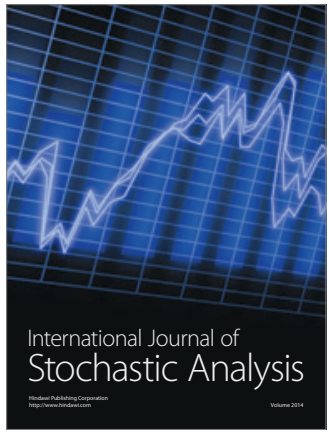

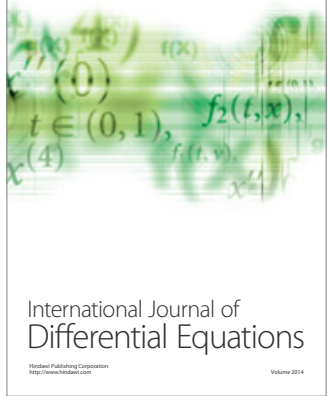
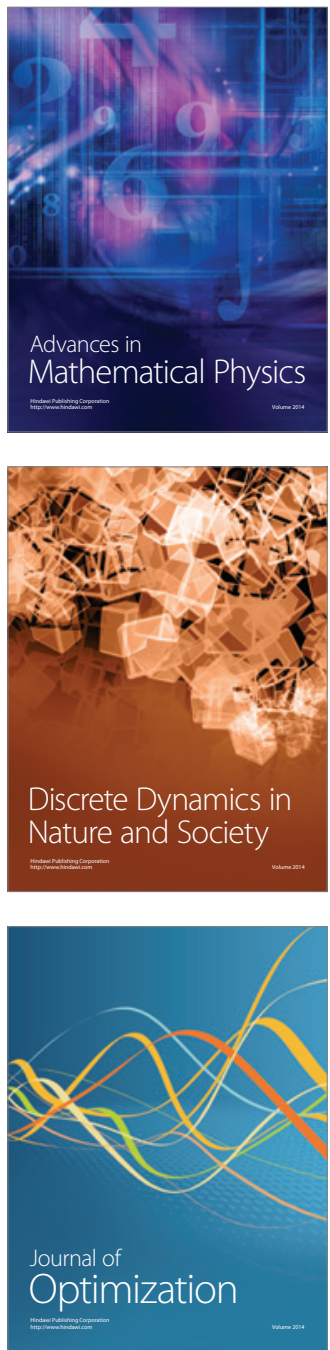